\title{
O uso do conceito paramétrico aplicado a uma inovação no mobiliário urbano: estudo de caso bicicletário
}

\author{
The use of parametric concept applied to an innovation in urban furniture: a case study bike \\ rack
}

\author{
Luciano Santos da Silva \\ Laboratório de Design e Seleção de Materiais \\ (LDSM) - Universidade Federal do Rio Grande do \\ Sul/UFRGS, Brasil \\ luciano.santos.silva@ufrgs.br

Gabriel Barbieri
Laboratório de Design e Seleção de Materiais
(LDSM) - Universidade Federal do Rio Grande do
Sul/UFRGS, Brasil
gabrielbarbieri22@gmail.com

\author{
Underléa Miotto Bruscatto \\ Departamento de Arquitetura (ARQ) - Universidade \\ Federal do Rio Grande do Sul/UFRGS, Brasil \\ arq.leiab@gmail.com
}

\author{
Fabio Pinto da Silva \\ Laboratório de Design e Seleção de Materiais \\ (LDSM) - Universidade Federal do Rio Grande do \\ Sul/UFRGS, Brasil \\ fabio.silva@ufrgs.br
}

\begin{abstract}
The concept of parametric design combines software and 3D modeling application that provides to designers, architects and engineers a new method for design development. This article aims to create a device for bicycle parking aided by a parametrization process using Grasshopper plug-in. Thus, we develop an algorithm in which its parameters can be modified accordingly to the esthetic-formal configuration required by the project. In order to evaluate the effect of a parametric value over the structure and resulting form, a rendering is created with each parameter change to visualize the resulting design interactively.
\end{abstract}

Keywords: Generative Design; Parametrization; Grasshopper Plug-in.

\section{Introdução}

A evolução dos programas computacionais facilitam e auxiliam o desenvolvimento de projeto. O design generativo é uma das ferramentas que possibilitam o desenvolvimento projetual em um nível elevado ao utilizar recurso CAD (Computer Aided Design) do qual permite parametrizar um projeto. Essa tendência, em constante evolução, permite criar desde formas simples a formas complexas, por meio da inserção de dados e parâmetros no projeto. Muitos projetos são gerados nessa tendência projetual: simulações de propriedades físicas (ZHU et al 2016); modelagem interativa digital do corpo humano aplicado ao desenvolvimento de produtos (BAEK et al., 2012); interpolação paramétrica (JIN et al., 2014); otimização de processos de usinagem (ZHOU et al., 2015) design de joias (STAMATI et al., 2004a 2011b); a parametrização de superfícies NURBS se mostra como uma possível direção para a computação e também uma importante ferramenta para o design de produtos (HUE et al., 2015; STAVRIC et al., 2011) e de projetos arquitetônicos (ZBOINSKA, 2015).

O design generativo é um processo construído por meio de regras e algoritmos, geralmente baseado em parâmetros. $O$ projetista interage com um sistema de configuração baseada em definições abstratas capazes de gerar inúmeras opções de projeto. Ao gerar diferentes alternativas com uma mesma linguagem, torna-se possível produzir famílias ou gerações de produtos. Possibilita também selecionar os melhores projetos entre as opções geradas que podem ser utilizados em diferentes produtos da mesma marca, por meio da modificação de elementos, de atributos e de parâmetros (FISHER \& HERR, 2001), ou seja, permite projetar, criar variações e escolher o melhor resultado.

Os sistemas generativos são executados mediante a uma linguagem existente ou a ser criada para estabelecer uma sequência de regras formais, aplicada de acordo com objetivo do projeto. Utilizam-se duas estratégias de linguagem: a gramática da forma e os algoritmos. A gramática da forma baseia-se no mecanismo de produção de frases da linguística, na qual os projetos são gerados a partir de um conjunto de regras pré-estabelecidas. $\mathrm{E}$ o algoritmo consiste em regras formais que servem para sistematizar e trazer a ordem ao processo de projetar, promovendo o aumento da produtividade e informações sobre as propriedades das formas ao designer (STINY \& GIPS, 1971; PRATTS, 2007).

O processo construtivo do design generativo permite o uso de ferramentas digitais para a construção de um modelo 3D computacional, conhecido como parametrização ou design paramétrico, ou seja, um método de projeto auxiliado por softwares. De forma ampla, a parametrização consiste em desenvolver um processo de comportamento rígido com informações estruturadas. Qualquer entrada de informações durante a sessão de desenho pode ser considerada como um parâmetro, a fim de produzir variações no desenho (HEU et al., 2015; CHRONIS et al., 2012; CELANI et al., 2011). 
Entretanto, o design generativo é compreendido por um método computacional que não se destina à projeção final de um objeto, mas à projeção de um sistema que por si desenha o objeto, permitindo gerar uma série de variações geométricas. Este processo permite trabalhar a genética de um objeto, ou sistema ao invés de modular apenas a sua forma final (ESTEVEZ, 2003). Dessa forma, visto que um dos recursos do design generativo permite o desenvolvimento de ações por meio de softwares em CAD, foi pensado na utilização do software de modelagem Rhinoceros e o plug-in Grasshopper, para a construção de um projeto paramétrico. $\mathrm{Na}$ interface do Rhinoceros, o software, permite o desenvolvimento de curvas, geometrias e superfícies (MCNEEL, 2014). O plug-in Grasshopper, que é um programa de linguagem virtual, baseado em um editor em nó, permite a criação de algoritmos generativos e da reprodução da geometria tridimensional (SANTOS 2014). O plug-in combina a programação visual com a programação textual dentro do mesmo ambiente, e dessa forma possibilita a parametrização de alternativas de design (KHABAZI, 2012). É possível ao editar uma combinação de dados no plug-in e visualizar em tempo real no software o resultado.

A proposta foi desenvolvida como estímulo nas atividades do Programa de Pós-Graduação em Design da UFRGS na disciplina de Fabricação Digital como processo de projeto, que propõe analisar as etapas de projeto no desenho industrial, por meio de ferramentas de fabricação digital e conceitos de desenho paramétrico.

Para o desenvolvimento desse trabalho, foi pensado em um elemento que necessitava de uma melhoria estética e funcional. Dessa forma, o elemento motivador foi identificado por meio da observação da dificuldade dos usuários em acomodar sua bicicleta nos espaços destinados aos ciclistas dentro da Universidade Federal do Rio Grande do Sul (UFRGS), ainda como a carência de aspectos estéticos e funcionais destes bicicletário. Foi pensado em um projeto paramétrico do qual fosse possível adicionar dados e alterar a estrutura formal do projeto. A interação do projeto com 0 software e plug-in resulta em um modelo digital de um bicicletário para uso interno da UFRGS. Ainda, foi feito um modelo digital, simulando o produto final.

\section{Métodos}

O objetivo principal da parametrização é desenvolver um produto de mobiliário urbano, especificamente um bicicletário, a fim de acomodar bicicletas na área interna da UFRGS. Para a geração e seleção da melhor alternativa de design, foi utilizado como ferramenta criativa o software de modelagem Rhinoceros e o plug-in Grasshopper. Para avaliar a melhor proposta utilizaram-se alguns algoritmos pré-definidos, de modo que existe a possibilidade de ajuste de dados a fim de desenvolver uma nova configuração de forma paramétrica. Para realização deste experimento foram aplicadas as orientações que constam em BROOM et al
(2015) e SFMTA (2011) como requisito conceituando o projeto. A recomendação é que os suportes de encosto devem permitir a fixação da bicicleta em dois pontos com altura mínima de $750 \mathrm{~mm}$.

As etapas de desenvolvimento projetual visaram identificar os requisitos do produto, e a partir da parametrização desenvolver um produto vinculado a esse aspecto. Sendo assim, o algoritmo gerado pelo software pode ser alterado com a inserção de dados, e dessa forma modificar o desenho em diversas formas, com a definição do produto digital.

Conforme apresentado por FLORIO (2014) no método de concepção de formas curvilíneas, para a orientação de superfícies regradas, com diretriz e geratriz gerada e manipuladas parametricamente para a criação de superfícies topológicas. Devido ao fato de ser um projeto de design de produto auxiliado por softwares, determinou-se um fluxograma de solução para o projeto (Figura 1), de uma forma simplificada visa resultados de design, da configuração paramétrica, bem como o auxílio proporcionado durante o processo de modelagem.

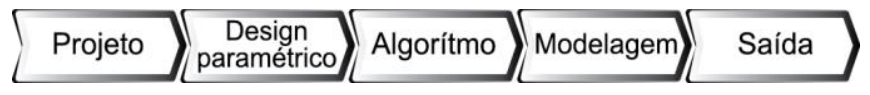

Figura 01 - Fluxograma projetual.

O funcionamento do fluxograma projetual permite o entendimento desde a concepção do projeto até a saída. $\mathrm{Na}$ etapa de projeto foram determinados parâmetros a partir das especificações indicadas por BROOM et al (2015) e SFMTA (2011), principalmente o controle da altura e os dois pontos de fixação do produto. O design paramétrico nesse projeto foi orientado para operações em CAD, proporcionando a criação do algoritmo. $O$ algoritmo permite a manipulação de valores que configuram uma nova alternativa de design, pode-se dizer que o algoritmo permite uma infinidade de alternativas, facilitando o estudo e entendimento formal e estendido até a escolha da melhor opção. Visto que o algoritmo permite a geração das informações projetuais em uma plataforma CAD a etapa de modelagem é facilitada, devido ao método estabelecido, e dessa forma, permite rápidos projetos, que podem ser renderizados ou enviados diretamente para um processo de fabricação digital, e conforme Celani (2008), as técnicas de fabricação podem ser: formativas, aditivas e/ou subtrativa. O uso da fabricação digital durante o projeto possibilita maior facilidade na criação de formas complexas, orgânicas e esculturais sem aumentos do tempo de produção ou custos (KAI \& FAI, 2004).

Assim, a partir desse fluxo, foi determinado o processo criativo para esse projeto. Que consiste em desenvolver uma estrutura paramétrica curvilínea da qual seja possível apoiar em dois pontos a bicicleta. Para isso, definiu-se um perfil 
básico conforme especificações de BROOM et al (2015) e SFMTA (2011). A partir do modelo projetado virtualmente foi possível modificar as curvas para confeccionar outros perfis visando um design paramétrico. As medidas limites definidas para esse módulo do bicicletário são: $1000 \mathrm{~mm}$ de largura, $800 \mathrm{~mm}$ de altura e um limite máximo de comprimento de $3000 \mathrm{~mm}$. Optou-se por um detalhamento do design paramétrico em todo o perfil. A interferência geométrica dada pelas modificações de parâmetros no algoritmo, não excede o padrão sugerido, dessa forma, não compromete a função, e permite uma melhora estética.

O método de parametrização no software Grasshopper foi inicializado pela definição de pontos no espaço, distantes por elemento de parametrização, construção de linhas pela ligação dos pontos, estas linhas formam o bloco virtual para descrever o estudo da superfície. Para a definição da superfície desejada essa foi identificada e seccionada em quatro curvas senóides pelo comando GraphMapper opção de seleção da curva tipo Sine (representa uma determinada função mapeada numericamente, no caso desse projeto foi utilizada o tipo seno) formando assim a superfície variável em forma de onda que servirá de referencia para secções do perfil dos módulos do bicicletário. Após a geração da superfície oriunda da função seno, esta foi seccionada por planos orientados na distância definida no projeto para acomodar um número de bicicletas no bicicletário. Gerando assim curvas que servem de referência para a orientação da curvatura dos tubos, formando o perfil do módulo.

O GraphMapper é uma interface de duas dimensões da qual permite modificar um conjunto de valores numéricos. A ferramenta funciona a partir de um valor de entrada que será lido no eixo $\mathrm{X}$ e mapeado usando a curva sobre o eixo $\mathrm{Y}$. Esta função é repetida em pontos determinados para gerar curvas que vão orientar a construção da superfície de orientação (principal) para o modelo do projeto.

Para a definição de alternativas desenvolveu-se um algoritmo onde dados são inseridos e podem facilmente manipular os resultados de design do produto. Isso devido a que 0 software considera ajustes pré-definidos com forma de padronizações para novas propostas de desenho. Aplicando diferentes parâmetros no algoritmo gerado, foram criadas diversas alternativas e selecionada a melhor. Ao escolher a melhor configuração, o modelo foi finalizado no software Rhinoceros, acrescentando as estruturas metálicas e renderizado para verificação do uso do produto.

\section{Resultados}

A partir das especificações elencadas nos métodos, o algoritmo foi desenvolvido no plug-in Grasshopper. A figura 2 mostra o princípio do desenvolvimento do algoritmo, que é detalhado da seguinte maneira: o início, parte da definição de um ponto na origem (A), este foi copiado e transladado no espaço (B), dessa forma os pontos foram unificados por uma linha (C), que é segmentada em partes iguais (D), formando assim a linha base do projeto.

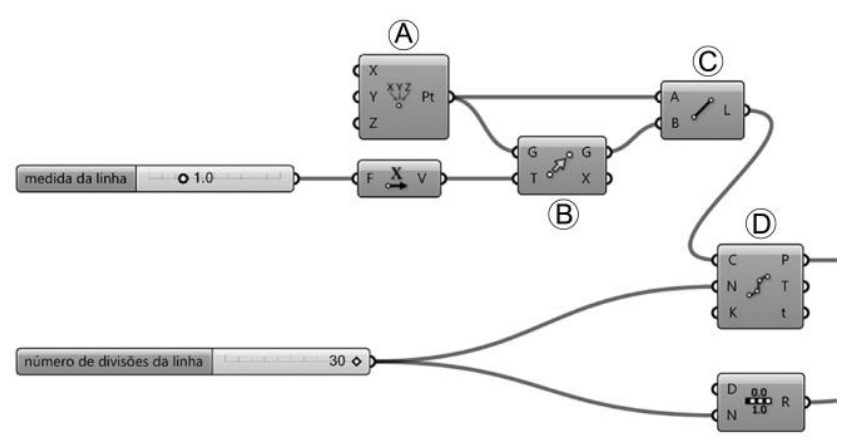

Figura 02 - Definição do ponto de origem e geração da linha

Tendo em vista a formação da curva, na figura 03 observa-se a sequência lógica do algoritmo, da qual foi criada uma lista de dados $(A)$, que serve para a orientação da curva senóide por meio do comando GraphMapper (B) com orientação para a curva seno. Os pontos da divisão da linha foram movidos no eixo $Z$ (C), construindo novos pontos no espaço, norteados pelo fator de amplitude e aplicados na curva formada pela função seno. Dessa forma que os pontos da divisão, são interpolados por uma linha (D). Esse procedimento foi repetido em quatro posições, para definir o limite do bloco virtual permitindo que cada lado seja modificado individualmente.

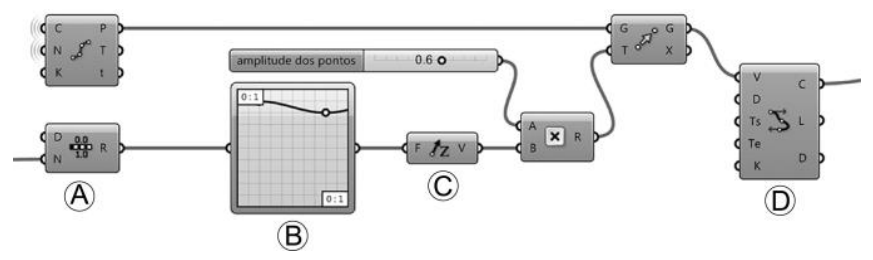

Figura 03 - Controle da curvatura por meio de parâmetros estabelecidos pelo comando GraphMapper

Determinado o controle da senóide, foram adicionados comandos que limitam as dimensões do produto. Assim, estabeleceu-se a altura de 10 a 1000 mm a largura em 10 a $1500 \mathrm{~mm}$ e o comprimento de 10 a $3000 \mathrm{~mm}$. Esses limitadores foram configurados por meio do posicionamento dos pontos no espaço, que unem os controladores da curva gerando delimitando o espaço de desenho virtual.

As quatro curvas resultantes da função GraphMapper, conforme observa-se na figura 4 , serviram para definir uma superfície principal, que foi construída por meio do comando loft (A). A partir disso, foram extraídas as arestas das curvas (B), separadas em listas de dados (C) e invertidas no sentido 
(D), resultando em oito linhas para formar as quatro superfícies (E).

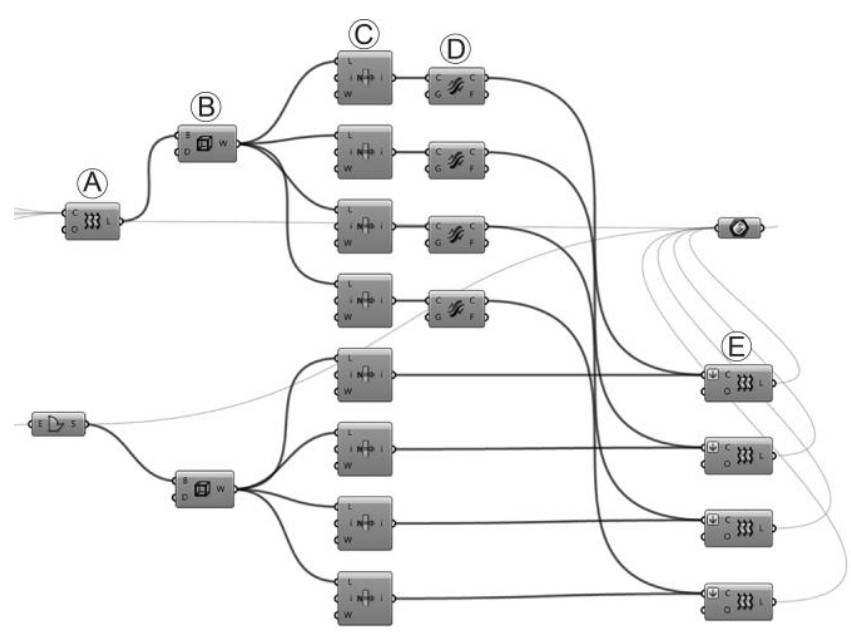

Figura 04 - Delimitação da superfície principal, e a programação para a função Freeform Loft e o fechamento da superfície por Loft.

Definidas as superfícies laterais do objeto a partir da função Loft, conforme mostra a figura 5 , o qual segue em uma orientação específica e fechando as laterais, foi utilizada a função $\operatorname{Brep}(A)$ que uniu as superfícies. As superfícies foram seccionadas com planos com normal no eixo $\mathrm{Y}$, distantes entre si pelo fator paramétrico (B). Além disso, foram criados pontos nas intersecções (C) que permitiram 0 arredondamento aleatório (D) das secções a partir de cada vértice, para uma breve análise foi simulado uma espessura de perfil com o comando offset $(E)$.

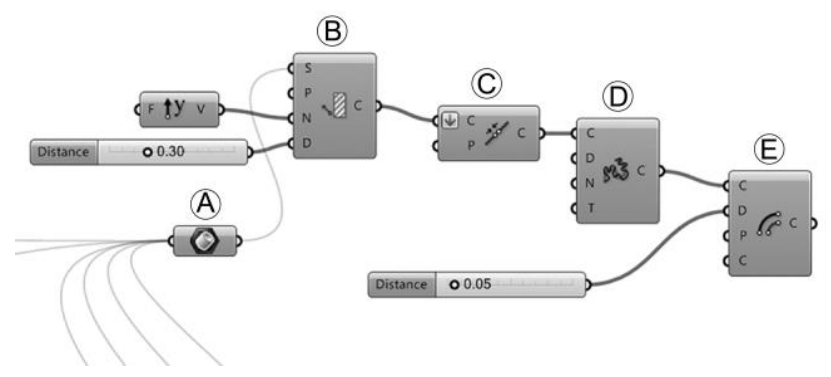

Figura 5 - (A e B) União e secção das superfícies. Em (C, D) arredondamento dos cantos das secções e (E) duplicação do perfil.

O resultado do algoritmo proporciona uma geração de diversas configurações de projeto. A alternativa escolhida, figura 6 , foi definida a partir das especificações dos prérequisitos determinados na metodologia. Com um módulo de $750 \mathrm{~mm}$ de altura e $1000 \mathrm{~mm}$ largura, o comprimento é definido por modulações individuais de $350 \mathrm{~mm}$ de distância entre os módulos, suficientes para o posicionamento de uma bicicleta.

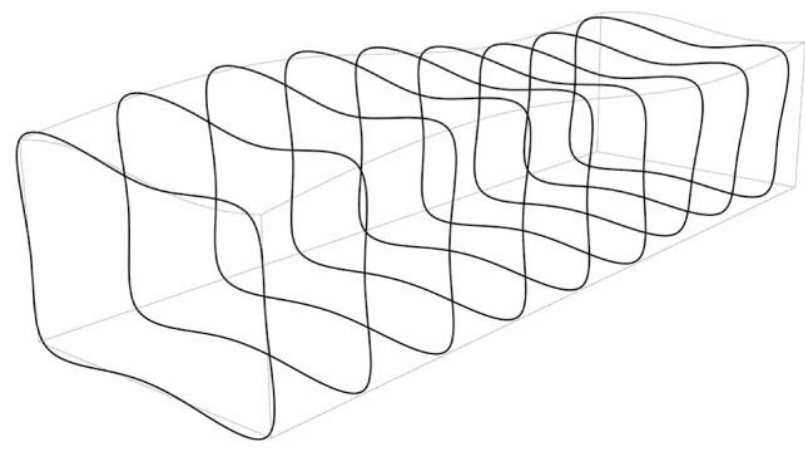

Figura 6 - Modelo da forma da secção do projeto definido a partir do algoritmo.

O material para a construção do bicicletário foi escolhido em aço de baixo teor de carbono com uma bitola padrão disponível no mercado de diâmetro de $32 \mathrm{~mm}$ e espessura de parede de $2 \mathrm{~mm}$. Esse material foi escolhido por ser de fácil manuseio e curvatura, tanto manual quanto mecânico. Foram inseridas barras de ligação entre os módulos e elementos de ligação para chumbamento no solo. O produto foi renderizado para simular o funcionamento e espaçamento entre módulos para acomodar as bicicletas, conforme mostra a figura 7 .
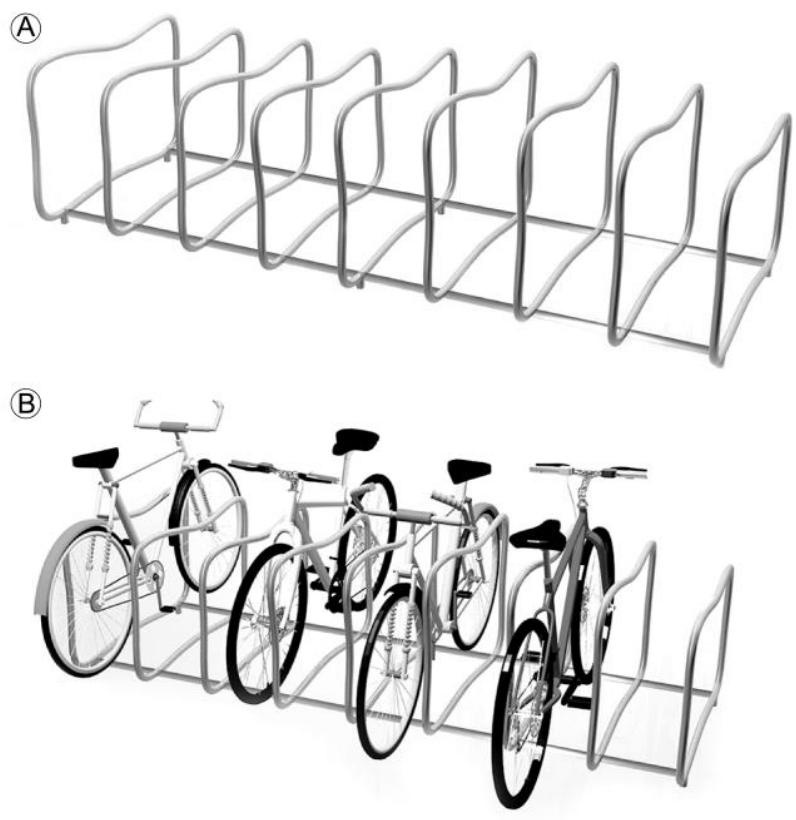

Figura 7 - Alternativa finalizada em rendering do produto $(A)$ e rendering do produto em uso (B). 


\section{Conclusões}

O desenho paramétrico orientado por softwares é uma ferramenta de grande auxílio para a geração de formas complexas no projeto de design ou de arquitetura. $O$ plug-in Grasshopper se destaca nesse meio devido à facilidade de desenvolver comandos que podem ser parametrizados por edição de parâmetros gerando modelos 3D no Rhinocerros. A ferramenta se mostrou potencialmente viável para a área de desenvolvimento de projetos.

Com a modificação de parâmetros específicos é possível à alteração de diversos aspectos na forma do produto. Por exemplo, de acordo com a demanda e com o espaço disponível, pode-se alterar o número de bicicletas suportadas pelo bicicletário. Isso acelera o processo de geração de alternativas fazendo com que a definição da melhor proposta seja rapidamente identificada.

Por ser um software vinculado à plataforma CAD proporciona fácil saída, principalmente para processos de fabricação digital como: usinagem, impressão $3 \mathrm{D}$, corte a laser. A proposta permite, que antes do envio do produto para a produção industrial, sejam feitas avaliações formais por processos mais rápidos permitindo a análise dimensional do produto e possibilidades de fabricação em larga escala, além de avaliar a configuração formal e estética a fim de determinar pontos positivos e negativos.

Assim, o design paramétrico auxiliado por softwares de programação, pode influenciar diretamente no desenvolvimento de produtos, propondo soluções de modo que o algoritmo calcule alterações e configure de forma rápida e precisa uma solução de projeto.

\section{Agradecimentos}

Agradecemos ao CNPq e ao CAPES, por incentivo ao aprimoramento, bem como o professor Wilson Florio, a Universidade Federal do Rio Grande do Sul e o Laboratório de Design e Seleção de Materiais.

\section{Referências}

BAEK, Seung-Yeob; LEE, Kunwoo.Parametric human body shape modeling framework for human-centered product design.Computer-Aided Design, v. 44, n. 1, p. 56-67, 2012. doi:10.1016/j.cad.2010.12.006

BROOM, N., ANDERSON, E., CARISTO, V., DODGE, R., DONLONWYANT, J., FIGLIOZZI, S., ... \& PATTERSON, B. Essentials of Bike Parking: Selecting and installing bicycle parking that works. (2015).

CELANI, G. VAZ, C.E.V. Scripts em CAD e ambientes de programação visual para modelagem paramétrica: uma comparação do ponto de vista pedagógico. Encontro de Tecnologia de Informação e Comunicação, v. 5, 2011.
CHRONIS, A.; LIAPI, K.; SIBETHEROS, L. A parametric approach to the bioclimatic design of large scale projects: The case of a student housing complex. Automation in construction, v. 22, p. 24-35, 2012.

FLORIO, W. Modelagem paramétrica, criatividade e projeto: dias experiências com estudantes de arquitetura. Gestão \& Tecnologia de Projetos, v.6, n.2, p. 43-66, 2012.

HSU, Ming-Chen et al. An interactive geometry modeling and parametric design plataform for isogeometric analysis. Computers \& Mathematics with Applications, 2015.

JIN, Yu-an et al. A fine-interpolation-based parametric interpolation method with a novel real-time look-ahead algorithm.ComputerAided Design, v. 55, p. 37-48, 2014.doi:10.1016/j.cad.2014.05.002

KHABAZI, Zubin. Generative Algorithms (using Grasshopper).Morphogenesism.2012.

LEE, Jae Yeol; KIM, Kwangsoo.Geometric reasoning for knowledgebased parametric design using graph representation.ComputerAided Design, v. 28, n. 10, p. 831-841, 1996.doi:10.1016/00104485(96)00016-4

MCNEEL, Robert. Rhinoceros 5. Manual del usuario para Windows. Robert McNeel\& Associates. 2014.

PIEGL, L. On NURBS: A Survey. IEEE Comput Graph Appl 1991:11(1):55-71. 2002

SANTOS, Nuno Miguel. Integração de Biónica em Design do Produto - Modelos de Design Generativo e Paramétrico em Estruturas Efêmeras. Dissertação Mestrado em Design de Produto. Faculdade de Arquitectura da Universidade de Lisboa. Lisboa, 2014.

SFMTA Municipal Transportation Agency. Bicycle Parking: Standards, Guidelines \& Recommendations (2011) Disponível em < https://www.sfmta.com> Acesso em 06/05/2015.

STAMATI, Vasiliki Antonopoulos G. Azariadis, P., \&Fudos, A parametric feature-based approach to reconstructing traditional filigree jewelry. Computer-Aided Design, v. 43, n. 12, p. 18141828, 2011.doi:10.1016/j.cad.2011.07.002

STAMATI, Vasiliki; FUDOS, Ioannis. A Parametric Feature-based CAD System for Reproducing Traditional Jewelry. ComputerAided Design and Applications, v. 1, n. 1-4, p. 559-567, 2004.doi:10.1016/j.cad.2004.07.004

STAVRIC, M.; MARINA,O. Parametric modeling for advanced architecture. International journal of applied mathematics and informatics, v. 5, n. 1, p. 9-16, 2011.

ZBOINSKA, Malgorzata A. Hybrid CAD/E platform supporting exploratory architectural design.Computer-Aided Design, v. 59, p. 64-84, 2015.doi:10.1016/j.cad.2014.08.029

ZHOU, Bo; ZHAO, Jibin; LI, Lun.CNC double spiral tool-path generation based on parametric surface mapping.ComputerAided Design, v. 67, p. 87-106, 2015.doi:10.1016/j.cad.2015.06.005

ZHU, Liangchao; LI, Ming; MARTIN, Ralph R. Direct simulation for CAD models undergoing parametric modifications.ComputerAided Design, 2016.doi:10.1016/j.cad.2016.05.006 